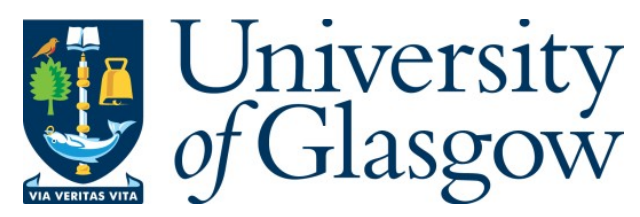

Gök, C. and Fuller, W. (2020) Topical review: shedding light on molecular and cellular consequences of NCX1 palmitoylation. Cellular Signalling, 76, 109791.

(doi: 10.1016/j.cellsig.2020.109791)

This is the Author Accepted Manuscript.

There may be differences between this version and the published version. You are advised to consult the publisher's version if you wish to cite from it.

https://eprints.gla.ac.uk/223527/

Deposited on: 25 September 2020

Enlighten - Research publications by members of the University of Glasgow http://eprints.gla.ac.uk 


\section{Topical Review: Shedding light on molecular and cellular consequences of NCX1 palmitoylation}

Caglar Gök, William Fuller*

Institute of Cardiovascular \& Medical Sciences, University of Glasgow

*: Correspondence will.fuller@glasgow.ac.uk, @FullerLabGlas

Sir James Black Building

University of Glasgow

University Avenue

Glasgow G12 8QQ

\section{Keywords:}

Sodium, calcium, microdomains, lipid rafts, palmitoyl acyl transferase, thioesterase, acylation, ion transport

\section{Abstract:}

Palmitoylation is the post-translational, covalent and reversible conjugation of a $16 \mathrm{C}$ saturated fatty acid to cysteine residues of proteins. The sodium calcium exchanger NCX1 is palmitoylated at a single cysteine residue in its large regulatory intracellular loop. Inactivation, mediated by the NCX1 inhibitory region XIP, is drastically impaired in unpalmitoylatable NCX1. The ability of XIP to bind and inactivate NCX1 is largely determined by NCX1 palmitoylation, which induces local conformational changes in the NCX1 intracellular loop to enable XIP to engage its binding site. Consequently, NCX1 palmitoylation regulates intracellular calcium by changing NCX1 sensitivity to inactivation. NCX1 palmitoylation is a dynamic phenomenon which is catalyzed by the palmitoyl acyl transferase zDHHC5 and reversed by the thioesterase APT1, with the switch between palmitoylated and depalmitoylated states, which has profound effects on NCX1 lipid interactions, influenced by NCX1 conformational poise. Herein we review the molecular and cellular consequences of NCX1 palmitoylation and its physiological relevance and highlight the importance of palmitoylation for NCX1 activity. We discuss the cellular control of protein palmitoylation and depalmitoylation, the relationship between lipid microdomains and lipidated and phospholipid binding proteins, and highlight the important unanswered questions in this emerging field. 


\section{Introduction}

Developing interest in palmitoylation and its physiological consequences in different tissues over the last decade has enabled palmitoylation to gain increasing popularity and put a spotlight on this unique post-translational modification. Simply put, palmitoylation is enzymatically controlled phenomenon involving the dynamic attachment of palmitic acid to a target protein via a thioester bond. This unique biochemical modification in protein structure to date, has been reported with a regulatory role in several cellular and physiological processes in various tissues [1-3]. Despite the rich background regarding the role of palmitoylation in the nervous system [3], how palmitoylation contributes to cellular events in the cardiac tissue; such as calcium homeostasis and cardiac contractility, is poorly understood.

Various ion channels/transporters and signalling molecules; which work in harmony as a team, lie at "the heart" of cardiac tissue. How this complex team functions to maintain cellular calcium fluxes in balance to support healthy cardiac function is vital question to be addressed. Among hundreds of proteins in the cardiac system, cardiac $\mathrm{Na}^{+} / \mathrm{Ca}^{2+}$ Exchanger (NCX1) is established as a major player in calcium homeostasis and cardiac contractility. NCX1 mediates $\sim 30 \%$ of cytosolic calcium removal in ventricles in large mammals [4]. NCX1 also acts as a part of rhythmic generator of action potential, the calcium clock, in the sinoatrial node (SAN) [5]. Inappropriate NCX1 behaviour is implicated in various cardiac pathologies with severe consequences [69].

NCX1 is 10 transmembrane (TM) domain protein with a large intracellular loop between TM5 and TM6. This intracellular loop is a key regulator of NCX1 function and is composed of Exchanger Inhibitory Peptide (XIP), Calcium Binding Domains (CBDs; CBD1 and CBD2) and the palmitoylation site (Figure 1) [10-16]. These regions promote different physiological states of the exchanger; XIP inhibits while calcium binding to CBDs activates the transporter [10,11,17-19]. Apart from the allosteric regulation of activation-inactivation states of NCX1 and the auto-regulation by XIP domain, palmitoylation is the first post-translational modification to be identified as a direct regulatory mechanism in NCX1 physiology [13-16, 20]. Although the discovery of NCX1 palmitoylation and its role in the exchanger function are relatively new to the field, the initial findings are promising for palmitoylation to be a powerful tool to tune NCX1 physiology. Herein we summarize physiological, molecular and cellular consequences of NCX1 palmitoylation updated with recent findings.

\section{Brief look up: What is currently known about NCX1 palmitoylation and its physiological consequences?}


NCX1 possesses multiple cysteine residues in its structure but exclusively only one palmitoylatable cysteine was detected at position 739 in the large intracellular loop of the exchanger (Figure 1) [13]. Abolishing NCX1 palmitoylation by introducing a single mutation at this site from cysteine to alanine (C739A) causes NCX1 to be unable to inactivate properly. Two separate experimental approaches using patch clamp in the whole cell configuration showed that a lack of palmitoylation disrupts the inactivation pattern of the exchanger. Chelating intracellular calcium with EGTA leads to the loss of calcium binding to the NCX1 CBDs. This intervention completely inhibited NCX1 current in the cells expressing wild type (WT) NCX1, but under the same conditions a significant portion of NCX1 current persisted in cells expressing unpalmitoylatable (C739A) NCX1. Similarly, the absence of intracellular ATP in the patch clamp experiments leads to depletion of PIP2 and release of the endogenous XIP domain to inactivate NCX1. PIP2 depletion caused $90 \%$ inhibition of wild type (palmitoylatable) NCX1 current but only inhibited $30 \%$ of the current when NCX1 is unpalmitoylatable [13]. So, timely and efficient inactivation of NCX1 requires palmitoylation of cysteine 739.

The extent of studies to understand the molecular basis of NCX1 palmitoylation uncovered an a helix structure adjacent to palmitoylated cysteine (C739) characterized with a large hydrophobic face and a small hydrophilic part (only 4 residues of the helical structure; D741, H745, T748 and K752) to control palmitoylation status of the exchanger (Figure 1) [15]. Deleting the entire amphipathic structure accommodated between 740 and 756 residues or manipulating either hydrophobic or hydrophilic face of the helix by mutagenesis drastically eliminated or diminished NCX1 palmitoylation (but did not affect the trafficking of NCX1) [15]. Precisely how this structural element governs NCX1 palmitoylation still remains unclear. It is likely recognised by the cellular palmitoylation machinery.

\section{Fresh from the oven: Recent advances on molecular and cellular importance of NCX1 palmitoylation}

3.1. Palmitoylation induces conformational changes in the intracellular loop of NCX1

The existence of an NCX1 dimer in plasma membrane is well-established [21]. The functional importance of NCX1 dimerisation is currently uncertain, but restructuring of the dimer appears to have important consequences for NCX1 regulation. Calcium dependent structural re-arrangements within the large intracellular loop of the exchanger were probed in oocyte membrane sheets using FRET sensors generated by insertion of either YFP or CFP to full length NCX1 at position 266, immediately after the XIP domain in the exchanger's large regulatory intracellular loop [12]. Binding of 
calcium ions to CBDs, which activates NCX1, promotes conformational changes in NCX1 dimers and reduces the distance between NCX1-NCX1 FRET pairs. In a recent investigation we probed the impact of palmitoylaton on the NCX1-NCX1 dimer using FRET measurements in live cells. We found that enhancing palmitoylation of NCX1 by supplementing with palmitic acid (PA) increased NCX1-NCX1 FRET, while reducing NCX1 palmitoylation with the palmitoylation inhibitor 2-bromopalmitate (2-BP) or complete loss of NCX1 palmitoylation by mutating the palmitoylated cysteine to alanine (C739A) reduced NCX1-NCX1 FRET [22]. This data suggest that palmitoylation either (1) changes the ability of NCX1 to dimerize or (2) triggers a local conformational changes in the existing NCX1 dimer. With the help of cross-linking approach using bismaleimidohexane $(\mathrm{BMH})$, the monomer/dimer ratio between palmitoylatable and unpalmitoylatable NCX1 was found to be identical [22]. It is now clear that palmitoylation restructures the large intracellular loop of the exchanger.

A note of caution should be sounded on the specificity of the palmitoylation inhibitor 2BP, used in these studies. Something of a 'workhorse' reagent for the palmitoylation field, the mixed pharmacology of 2-BP is extensively documented, but frequently ignored [23]. Cell convert 2-BP to its CoA ester, which irreversibly inhibits zDHHCPATs by modifying their active site [24]. However, 2-BP was established to inhibit mitochondrial fatty acid oxidation nearly 50 years ago [25], and recent studies using 2BP analogues have identified numerous cellular targets other than zDHHC-PATs for this very promiscuous reagent [26, 27], including the depalmitoylating enzymes themselves [28]. Unfortunately, the field is yet to identify a zDHHC-PAT inhibitor with a more reliable pharmacology. Given the concerns about its specificity, we suggest 2BP should only be employed alongside genetic approaches (cysteine to alanine mutations, zDHHC-PAT silencing) to probe the functional consequences of protein palmitoylation.

\subsubsection{What does NCX1-NCX1 FRET tell us about physiology and ion transport?}

From the point of view of NCX activity, it is important to consider what the high FRET and low FRET forms of NCX1 actually represent, and what regulatory mechanisms might be signposted for future investigation? The FRET experiments by John and colleagues that concentrated on calcium dependent re-structuring of NCX1 dimers reported increased FRET signals between existing NCX1-NCX1 dimers following NCX1 activation by $\mathrm{Ca}^{2+}$ binding to CBDs [12]. Mutations within CBD1 abolished $\mathrm{Ca}^{2+}$ dependent structural changes within the intracellular loop of the exchanger detected using FRET. On the other hand, mutation of CBD2 did not affect NCX1-NCX1 FRET behaviour but did impair calcium regulation. This indicates that the $\mathrm{Ca}^{2+}$-induced dimer 
movement between NCX1 pairs measured by NCX1-NCX1 FRET occurs mainly as a result of $\mathrm{Ca}^{2+}$ binding to CBD1. One might question the ability of FRET sensors positioned at 266 to detect CBD2-driven structural rearrangements. Clearly, CBD1 is situated nearer the sensors, which may enhance their ability to detect any restructuring of this region. However, the fact that palmitoylation-dependent changes in NCX1-NCX1 FRET can be detected even though the palmitoylation site is further from the FRET sensors than CBD2 suggests that the sensors are positioned appropriately to detect restructuring throughout the NCX1 intracellular loop.

As for our understanding of what the impact of palmitoylation on NCX1-NCX1 FRET means for NCX1 itself, several intriguing possibilities exist. The fact that the palmitoylation-dependent change in NCX1-NCX1 FRET requires intact lipid rafts (see section 3.2) clearly implicates changes in phospholipid interactions with the exchanger driven by palmitoylation. We need to understand the identities and regulatory role of these phospholipids. However, another significant effect of palmitoylation on NCX1 is to enhance its affinity for the inhibitory XIP domain (see section 3.5). It may be that we can marry these observations of enhanced NCX1-NCX1 FRET and enhanced NCX1 inactivation if inactivation by XIP occurs "in trans" between NCX1 dimers. In other words, perhaps the FRET change induced by NCX1 palmitoylation reports the changes in distance between the XIP domain in one protomer and its binding site on the second.

\subsection{Palmitoylation of NCX1 modifies its affinity for and the behaviour of lipid microdomains}

The physical mechanisms underlying partitioning of proteins into lipid microdomains are the subject of ongoing investigations. Indeed, it is likely that numerous mechanisms, including cholesterol binding motifs, transmembrane domain physical properties and tertiary structure, and the presence of lipid modifications control microdomain affinity [29-32]. Investigations in Giant Plasma Membrane Vesicle (GPMV); a model system that is cell-detached structures (Figure 2A) [33-36], have elucidated an important role for palmitoylation to enhance the affinity of proteins for the raft (ordered) phase of isolated membranes which is rich in saturated phospholipids, cholesterol, glycolipids and sphingolipids [31, 37]. For example, the transmembrane linker for activation of T cells (LAT) clustering in the lipid rafts is markedly dependent on its palmitoylation status at two juxtamembrane cysteines [31].

Since a typical GPMV sustains the lipid and protein composition of the native membrane, it is an ideal and sophisticated model to look into protein distribution through lipid microdomains. We induced phase separation in GPMVs to unravel how 
palmitoylation impacts the interaction of the exchanger with lipid microdomains, and found that loss of palmitoylation remarkably prompts the localization of NCX1 almost entirely within the non-raft (disordered) phase enriched with phospholipids incorporating unsaturated fatty acids (Figure 2) [22]. Curiously, although palmitoylated NCX1 showed a significantly greater overlap with lipid raft markers than nonpalmitoylated NCX1 (Figure 2D), the ability of GPMVs to separate into raft and nonraft phases was impaired by the presence of palmitoylated NCX1. The overlap of raft and non-raft markers was significantly greater when NCX1 was palmitoylated than when it wasn't (Figure 2C). This implies a direct effect of palmitoylated NCX1 on lipid behaviour within GPMVs, a phenomenon for which other precedents are emerging. For example, the integral membrane protein PMP22 has recently been found to enhance phase separation of GPMVs and alter the circumference of the GPMVs in which it resides, confirming that multi-pass integral membrane proteins can alter the biophysical properties of membranes in which they reside [32]. Indeed NCX1 and PMP22 represent the first examples of multi-pass integral membrane proteins to show a significant affinity for the ordered phase of GPMVs - albeit via very different mechanisms.

So how does palmitoylated NCX1 exert an influence on GPMV phase separation? Since the XIP domain of NCX1 binds PIP2, it is tempting to speculate that the recruitment of this lipid species (which is usually confined to the non-raft phase in GPMVs) conflicts with an enhanced affinity of palmitoylated NCX1 for raft phase lipids, impairing phase separation and effectively lowering the temperature at which phase separation can be achieved. This is similar to molecular dynamics simulations of the partitioning of palmitoylated and farnesylated $\mathrm{H}$-Ras, in which the saturated palmitate attracts $\mathrm{H}$-Ras to the lipid ordered phase, but the unsaturated farnesyl attracts it to the disordered phase. As a result, $\mathrm{H}$-ras is thought to cluster at order / disorder boundaries [38]. In our experiments, the ability of NCX1 to bind the unsaturated lipid PIP2 may generate the same outcome in GPMVs. We therefore suggest a similar impact on the partitioning of a protein between ordered and disordered phases for a multi-pass integral membrane protein (via PIP2 binding and dynamic palmitoylation) as has been established for peripheral membrane proteins (via palmitoylation and farnesylation). A similar phenomenon has also recently been postulated for the dually palmitoylated PIP2 binding protein CD44. Palmitoylation recruits CD44 into the raft phase, while PIP2 can extract singly but not doubly palmitoylated CD44 from rafts [39]. Additional complexity in this system is generated by the unequal contributions of the two palmitoylation sites to CD44 raft affinity. The CD44 cytosolic palmitoylation site presents its palmitoyl chain perpendicular to the membrane normal, which allows it to 
insert parallel to the phospholipid tails. In contrast, the palmitoyl chains attached to the CD44 integral membrane palmitoylation site are forced to adopt an unfavourable angle relative to the bilayer. In other words, simply being palmitoylated isn't the only thing to consider with respect to lipid raft localization; the distance of a palmitoylation site from a transmembrane domain will also impact raft affinity of an integral membrane protein. Why is this so important for the cell? Many other palmitoylated peripheral and integral membrane proteins also bind PIP2. Ultimately then, palmitoylation of a lipid anchored or lipid binding protein may remodel the local membrane environment and influence binding of regulatory lipid species as well as modifying the structure and function of the target protein itself, with important implications for cell physiology.

Supporting the data gathered in GPMV experiments, remodelling the plasma membrane in intact cells with methyl- $\beta$-cyclodextrin (M $\mathrm{BCD}$ ); to deplete cholesterol acutely, or SDS; to promote increased raft formation, modifies NCX1-NCX1 FRET only when it is palmitoylatable. Much like 2-BP discussed above, the use of M $\beta C D$ to deplete cholesterol and destroy membrane rafts is frequently criticised as a blunt tool with significant potential for off-target effects [40]. This reagent must be used cautiously as part of an integrated repertoire of approaches to understand membrane raft roles and behaviours. Cholesterol depletion in the intact cells expressing WT-NCX1 reduced FRET between NCX1 pairs, but NCX1-NCX1 FRET was increased when raft formation was enhanced by SDS treatment. Neither MBCD nor SDS treatment affected FRET behaviour of unpalmitoylatable (C739A) NCX1 [22]. So, when NCX1 cannot be palmitoylated, it cannot recognize and interact with the raft phase. These findings suggest a new notion that raft formation or the presence of particular phospholipid species might be a factor facilitating or even required for palmitoylation-induced restructuring of the NCX1 intracellular loop. This may explain why manipulating lipid composition of plasma membrane in intact cells with M $\beta C D$ and SDS affected NCX1NCX1 FRET only in palmitoylatable NCX1; more rafts $=$ high $\mathrm{FRET}$ and less rafts $=$ low FRET. To conclude, palmitoylation of NCX1 is a key process for appropriate interaction with lipids.

\subsection{Allosteric regulation and NCX1 palmitoylation}

Ion transport by NCX1 is an entirely thermodynamically driven event, but it can be facilitated or inhibited by allosteric regulation. This concept of allosteric regulation of NCX1 was originally identified over 3 decades ago by Kimura and colleagues [41]. NCX1 is allosterically activated by increased cytosolic calcium binding to the CBDs, which increases the rate of ion exchange across the membrane. Elevated cytosolic sodium allosterically inactivates NCX1 in a process usually referred to as sodium- 
dependent inactivation [42]. We found that FRET between NCX1 pairs differs in response to different extracellular buffers; Tyrode's, $\mathrm{Na}^{+}$free- and $\mathrm{Ca}^{2+}$ free- buffer. Compared to recordings made in Tyrode's, exposing cells expressing WT-NCX1 to $\mathrm{Na}^{+}$containing, $\mathrm{Ca}^{2+}$ free extracellular solutions enhanced NCX1-NCX1 FRET, while $\mathrm{Ca}^{2+}$ containing, $\mathrm{Na}^{+}$free buffers reduced the FRET signal between NCX1 pairs. Of course, allosteric regulation has long been known to put NCX1 through conformational changes to adapt to distinct ionic conditions. However, in FRET experiments, unpalmitoylatable NCX1 responded to the changes in extracellular environment differently from WT-NCX1: the FRET signal of unpalmitoylatable exchanger was enhanced in $\mathrm{Ca}^{2+}$ containing, $\mathrm{Na}^{+}$free conditions, but there was no change in FRET signals in $\mathrm{Na}^{+}$containing, $\mathrm{Ca}^{2+}$ free extracellular solutions. We also noted a striking increase in NCX1 palmitoylation in the cells incubated in $\mathrm{Ca}^{2+}$ free buffer and a decrease in $\mathrm{Na}^{+}$free buffer, suggesting that a cycle between palmitoylated and depalmitoylated state of NCX1 is promoted by distinct ion-bound structures [22].

Not only does this imply that palmitoylation and depalmitoylation of NCX1 occurs dynamically in the cell (see section 3.4), but it also suggests that the palmitoylation machinery can distinguish between the structures adopted by NCX1 when it is allosterically regulated. The enhanced FRET signal of unpalmitoylatable NCX1 in $\mathrm{Ca}^{2+}$ containing, $\mathrm{Na}^{+}$free conditions may represent the previously-established $\mathrm{Ca}^{2+}$ dependent FRET changes induced by $\mathrm{Ca}^{2+}$ binding to CBD1 [12]. However, this modest increase in FRET is dwarfed by a very substantial decrease in FRET under the same conditions when NCX1 can be palmitoylated. Meanwhile the enhanced NCX1 palmitoylation and FRET observed in $\mathrm{Na}^{+}$containing, $\mathrm{Ca}^{2+}$ free conditions suggests a positive feedback loop in which sodium binding to its allosteric regulatory site (already established to promote NCX1 inactivation) enhances NCX1 palmitoylation, further sensitizing it to inactivation by XIP. Hence palmitoylation appears to be an important feature of the allosteric regulatory mechanisms for NCX1.

\subsection{Palmitoylation of NCX1 is catalysed dynamically at cell surface by zDHHC5 and} reversed by APT1

The palmitoylation/depalmitoylation cycle is an enzymatically controlled process. Catalysis of protein palmitoylation involves protein-acyltransferases (PATs), a large family with twenty three isoforms. These integral membrane proteins typically have 4 transmembrane domains, with the catalytic site within a unique cysteine rich, zinc finger containing motif: Asp-His-His-Cys (zDHHC) [24, 43]. Most zDHHC-PATs are found at a single (sometimes two) location(s) within the secretory pathway, with the Golgi apparatus widely recognised as the cellular palmitoylation 'hub' [44-47], and 
relatively few zDHHC-PATs responsible for dynamic palmitoylation of proteins residing at the cell surface. Substrate recognition rules and upstream control of zDHHC-PAT activity is an emerging area of research. Some zDHHC-PATs display high activity but relatively low substrate affinity and specificity $[48,49]$. Others, among them zDHHCs 5,13 and 17 , form stable interactions with their substrates, making them amenable to affinity purification techniques [49, 50]. Regulation of cardiac excitability and contractility by dynamic palmitoylation of ion transporters has been discussed in detail by others [51]. Briefly, zDHHC-PATs control cardiomyocyte excitability by regulating the voltage gated sodium channel SCN5a [52], active transport by the Na/K ATPase via its accessory protein phospholemman $[49,53,54]$, action potential duration by the potassium channel Kv4.3 via its accessory protein $\mathrm{KChiP2}[55,56]$, calcium influx via the beta subunit of the L-type Ca2+ channel $[57,58]$, as well as contractility via phospholamban [59] and NCX1. Until very recently, of the cell surface ion transport regulators it was only phospholemman that had been 'paired' with a palmitoylating enzyme, the cell surface zDHHC-PAT zDHHC5 [49, 50, 60].

Compared to the large family of PATs identified in yeast $[61,62]$ and mammals [6368], our knowledge about depalmitolating enzymes is limited with the discovery of acylprotein thioesterases (APTs; APT1 and APT2) [69-72], lysosomal palmitoyl-protein thioesterases (PPTs; PPT1 and PPT2) [73-75] and recently identified FASN, PNPLA6, ABHD6, ABHD16A, ABHD17A/B/C [76].

\subsubsection{The NCX1 depalmitoylating enzyme, APT1}

Detailed mutagenesis scanning on NCX1 structure has brought a deep understanding of structural elements involving in palmitoylation of NCX1. As aforementioned, the ahelix structure that sits between residues 740 and 756 is a key structural element for NCX1 palmitoylation. Hypothesising that this helical structure interacts with the enzyme(s) involved in the NCX1 palmitoylation/depalmitoylation process, the heart and brain interactomes of a biotin-tagged peptide which corresponds to this helix were idenitfied using LC-MS/MS. Notably, the only palmitoylation/depalmitoylation processes related protein which was pulled out by this peptide was APT1. Further experimental approaches using pharmacological tools targeting APT1 and APT2 confirmed that APT1 but not APT2 reverses NCX1 palmitoylation [22]. The relatively non-specific thioesterase inhibitor Palmostatin B and the APT1-specific inhibitor ML348 both increased steady state NCX1 palmitoylation when applied to HEK cells and adult ventricular myocytes. In contrast, the APT2-specific inhibitor ML-349 did not alter NCX1 palmitoylation in either cell type. 
The original investigations of the depalmitoylating activity of APT1 (also known as LYPLA1) concluded that this enzyme was cytosolic [70], a finding that was replicated in many follow-up studies. In contrast, a recent investigation suggested that the majority of APT1 is localised in mitochondria [77]. In live cells, mitochondrially-targeted fluorescence-based activity probes engineered to detect cysteine deacylation activity demonstrated the presence of active depalmitoylating enzymes in mitochondria. Subsequent siRNA and pharmacological screens suggested that a substantial fraction of the mitochondrial deacylation activity detected by these probes was due to the presence of APT1 in mitochondria, while ATP2 was found in the cytosol and at the Golgi apparatus. Myc-tagged APT1 localised to mitochondria while fluorescent protein APT1 fusions did not, and endogenous APT1 copurified with mitochondrial markers in fractionation experiments (although in the same experiments a small fraction of endogenous APT1 was found outside mitochondria) [77]. It is difficult to reconcile these observations with the very many papers that rigorously demonstrate that APT1 is cytosolic in eukaryotes from yeast to mammals [70-72, 78-81]. The ability of APT1 to depalmitoylate non-mitochondrial targets such as heterotrimeric and small $G$ proteins is well accepted [82]. Indeed, a recent quantitative palmitoyl-proteomics screen of APT1 knockout endothelial cells identified 52 candidate APT1 substrates, none of which are annotated as mitochondrial proteins [83]. So, this enzyme appears to live a dual life. A small (but from a palmitoylation perspective the functionally significant) population depalmitoylates non-mitochondrial proteins in the Golgi apparatus and at the plasma membrane - including NCX1. A second pool resides in mitochondria. The identity of the mitochondrial APT1 substrates remains elusive, raising the possibility that mitochondrial-localised APT1 either has no unique substrates, or performs an enzymatic function distinct from depalmitoylation (for example, hydrolysis of long chain mono-acyl glycerol esters [84]). To date, only the recently-identified depalmitoylating enzyme ABHD10 has been established to depalmitoylate a mitochondrial substrate the redox sensitive protein peroxiredoxin 5 [85].

\subsubsection{An NCX1 palmitoylating enzyme, zDHHC5}

Surprisingly, our proteomics experiments identified no zDHHC-PATs in the affinity purification experiments using the palmitoylation-directing NCX1 a-helix. This might be due to the limitation in the specificity/detection capability of LC-MS/MS. In our extensive experience detecting integral membrane proteins using mass spectrometry $[50,86]$, zDHHC-PATs are only rarely observed, suggesting they are relatively low copy number proteins. In a separate experiment we evaluated a relationship between NCX1 and zDHHC5, a cell-surface located zDHHC-PAT [87] which recognizes its 
substrates via its extended C tail [49]. Using a peptide library based on the zDHHC5 C-tail, we found zDHHC5 interacts with NCX1 [22]. In experiments in zDHHC5-KO cells, NCX1 was still palmitoylated, albeit to a lesser extent than in wild type HEK cells. Re-introducing zDHHC5 into zDHHC5-KO cells significantly increased NCX1 palmitoylation. This suggests that not only zDHHC5 but also other zDHHC-PATs catalyse NCX1 palmitoylation. FRET between NCX1 pairs in ZDHHC5-KO cells was not altered significantly by $\mathrm{Ca}^{2+}$ free conditions as opposed to those measured in wild type HEK cells [22], suggesting that the changes in NCX1 palmitoylation associated with allosteric regulation require the presence of $\mathrm{zDHHC5}$. These findings are important to understand the nature of palmitoylation/depalmitoylation of NCX1. On the other hand, the substantial residual palmitoylation of NCX1 in ZDHHC5-KO cells means that more experiments are needed to understand the full repertoire of zDHHCPATs that are capable of palmitoylating NCX1.

Interestingly, the region of the $\mathrm{ZDHHC5} \mathrm{C}$ tail that interacts with NCX1 is the same region that we recently established to interact with the Na pump / PLM complex via the pump's third intracellular loop. Functional and physical interactions between the $\mathrm{Na}$ pump and NCX1 are well established [88-91]; whether such interactions are relevant to $\mathrm{ZDHHC5}$ regulation of either transporter remains to be established. The region of zDHHC5 that interacts with the Na pump and NCX1 in cardiac muscle has also been implicated in adaptor protein interactions and ZDHHC5 substrate recruitment in other tissues $[92,93]$. Hence zDHHC5 selection of substrates may depend on the tissuespecific presence or absence of adapters facilitating substrate engagement. Such a phenomenon is not unique to $\mathrm{zDHHC5}$, as $\mathrm{zDHHC9}$ palmitoylation of $\mathrm{H}$-ras also relies on the presence of an adaptor protein [68]. However, the majority of zDHHC-PATs are thought to either engage directly with their substrates [24, 94], or to utilise recognition motifs integral to their $\mathrm{N}$ and / or $\mathrm{C}$ termini (for example ankyrin repeats [95]). Serendipitously, this unique complexity associated with substrate recruitment by zDHHC5 offers the opportunity for tissue-specific or substrate-specific targeting, once the molecular contact points between $\mathrm{ZDHHC} 5$ and its substrates have been identified [96].

The mechanisms underlying regulation of $\mathrm{ZDHHC5}$ substrate palmitoylation have been the subject of considerable interest recently. Palmitoylation of the zDHHC5 C tail by zDHHC20 increases Na pump recruitment and palmitoylation, while GlcNAcylation of a nearby serine does the same [50]. In the brain, zDHHC5 palmitoylation of $\delta$-catenin relies on its internalisation caused by synaptic activity-induced zDHHC5 dephosphorylation [97]. Adapter protein interactions are promoted by palmitoylation of the zDHHC5 C tail $[92,93]$. A common theme in the cellular regulation of zDHHC5 
therefore appears to involve post-translational modifications regulating substrate recruitment - with little evidence that the catalytic turnover of palmitate in the enzyme's active site can be modified. Whether ZDHHC5 recruitment and palmitoylation of NCX1 is regulated in the same manner remains to be seen. However, the fact that changes in NCX1 palmitoylation induced by allosteric regulation require the presence of zDHHC5 is suggestive of cellular mechanisms regulating zDHHC5 recruitment and palmitoylation of $\mathrm{NCX} 1$ that remain to be discovered.

The repertoire of substrates known to be palmitoylated by zDHHC5 therefore continues to expand. In epithelial cells $\mathrm{ZDHHC5}$ regulates cell adhesion via palmitoylation of desmoglein-2 and plakophilin-3 mediated by the adaptor protein Golga7b [93]. In multiple cell types a subtly different adaptor protein, Golga7, partners zDHHC5 in controlling non-apoptotic cell death [92]. Meanwhile in cardiac muscle zDHHC5 is emerging as a key regulator of intracellular $\mathrm{Na}$ via its ability to regulate two of the major cellular Na efflux routes, NCX1 and the Na pump via PLM [49, 50]. Given the central importance of intracellular $\mathrm{Na}$ for mitochondrial function [98-102], and the established defects in cellular sodium handling that underlie cardiac hypertrophy and heart failure [102-106], zDHHC5 regulation of cardiac Na handling is an area ripe for therapeutic exploitation.

\subsection{Palmitoylation dependent conformational changes within the intracellular loop of} NCX1 favors XIP binding and ultimately regulates intracellular $\mathrm{Ca}^{2+}$

The NCX1 auto-inhibitory mechanism facilitated by its endogenous XIP domain has been always compelling to fully understand NCX1 pharmacology. Deciphering the molecular nature of XIP dependent auto-regulation of the exchanger could potentially pave the way to innovate pharmacological strategies to ameliorate inappropriate NCX1 function in cardiac pathologies.

The interaction of auto-inhibitory peptide with the XIP docking site, identified as the residues between 562 and 679 [107], enables NCX1 to inactivate. We had previously established that NCX1 must be palmitoylated in order to switch to the inactive state [13], and recently extended this observation to demonstrate that palmitoylation creates a space for XIP to bind NCX1 [22]. Disruption of NCX1 palmitoylation using 2-BP or by mutating the palmitoylated cysteine to alanine significantly decreased the relative amount of NCX1 affinity-purified by a biotinylated XIP peptide [22]. The region between NCX1 residues 562 and 679 defined as the XIP docking site includes part of CBD2 and is considerably larger than XIP itself, which is only 25 amino acids. We hypothesised that the XIP binding site was considerably smaller. Employing peptide affinity purification and peptide array scanning approaches, we identified the precise 
binding site as residues 709-728, positioned close to NCX1 palmitoylation site. Deletion of amino acid residues between 709 and 728 did not change the palmitoylation status of NCX1, but led complete loss of XIP binding to NCX1 [22]. The implication of this finding is that the conformational changes within the intracellular loop of NCX1 initiated by palmitoylation create a binding site for the XIP domain just on the $\mathrm{N}$ terminal side of the palmitoylation site [22]. The insensitivity of non-palmitoylated NCX1 to inactivation, and reduced affinity purification of non-palmitoylated NCX1 by a biotinylated XIP peptide are both explained by this observation.

So what of the previous observation that the XIP docking site lies in the region between residues 562 and 679 of NCX1 [107]? It is important to remember that XIP is not merely an NCX1-antagonist: the actions of XIP are to promote formation of an inactivated form of NCX1 that is incapable of transporting ions. In some respects this inactivated form is diametrically opposed to the activated state induced by calcium binding to the CBDs. $\mathrm{XIP}$ is therefore better regarded as an 'inverse-agonist', in which the actions of binding / engagement and efficacy (inactivation) can be regarded as separate events. Our findings therefore imply that XIP binding is directly regulated by palmitoylation and requires residues 709-728, while XIP efficacy is indirectly regulated by palmitoylation (due to the effect on binding) and requires residues 562-679. There are important implications to this finding. Experimental strategies to antagonise the actions of XIP need only be targeted towards the XIP binding site. However, experimental or therapeutic strategies to enhance NCX1 inactivation may need to target both the XIP binding site as well as the region around CBD2 that is required for XIP efficacy. Antagonising XIP may therefore prove a much easier strategy than mimicking it. This will be the subject of future investigations.

\subsection{NCX1 palmitoylation and transmembrane $\mathrm{Ca}^{2+}$ flux}

Ultimately, by changing the sensitivity of NCX1 to inactivation by XIP, palmitoylation regulates transmembrane $\mathrm{Ca}^{2+}$ flux through $\mathrm{NCX} 1$ and hence intracellular $\mathrm{Ca}^{2+}$ levels. In cells engineered to express wild type NCX1, intracellular calcium is lower than in cells engineered to express unpalmitoylatable NCX1. Supporting this, increasing NCX1 palmitoylation in the cells by inhibiting its depalmitoylation with the APT1 inhibitor PalmB reduced the intracellular calcium concentration in the cells expressing WT-NCX1 [22]. It is noteworthy that in HEK cells (resting membrane potential $-24 \mathrm{mV}$ [108]), NCX1 acts as calcium influx pathway under physiological conditions (NCX1 reversal potential $-42 \mathrm{mV}$ ). In excitable tissues with a resting membrane potential more negative than the reversal potential $(-80 \mathrm{mV}$, cardiac myocytes), NCX1 is predominantly a $\mathrm{Ca}^{2+}$ efflux pathway. Ultimately then, by changing the sensitivity of 
NCX1 to inactivation, the palmitoylation status of NCX1 has the potential to control intracellular calcium load in multiple cell types. For example, in cardiac muscle any reduction in NCX1 palmitoylation will reduce the the propensity of NCX1 to inactivate, enhance NCX1-mediated $\mathrm{Ca}^{2+}$ efflux, and reduce sarcoplasmic reticulum calcium content. As well as having a direct effect on contractility, enhanced NCX1-mediated $\mathrm{Ca}$ efflux is pro-arrhythmic (because it generates an inward current) and increases intracellular $\mathrm{Na}^{+}$, with all the negative impacts on mitochondrial function previously discussed.

Putting all together, these results resolve an important question regarding what NCX1 inactivation stands for in the cell. The fact that NCX1 palmitoylation status influences intracellular $\mathrm{Ca}^{2+}$ (despite NCX1-mediated currents under tightly controlled intracellular conditions being unchanged by palmitoylation $[13,14])$ indicates that XIP-mediated 'tuning' of NCX1 activity is an important factor even in the absence of external stimuli. Recent findings thus bring a thorough mechanistic insight into physiological relevance of XIP driven NCX1 inactivation at molecular level and its modulation by palmitoylation.

\section{Conclusion \& Future Perspectives}

Recent findings highlighted here have brought a new dimension into the understanding of the role for palmitoylation in NCX1 function. NCX1 is dynamically palmitoylated at the cell surface by zDHHC5 and depalmitoylated by APT1. Palmitoylation is a key regulatory process for $\mathrm{NCX} 1$ inactivation and the control of intracellular calcium as well as for NCX1 interaction with lipid domains. However, the cellular events that control NCX1 palmitoylation and depalmitoylation as well as some aspects of the molecular nature of palmitoylation-complex still remain unclear. Indeed compared to posttranslational modifications such as phosphorylation, the palmitoylation field as a whole suffers from a relatively crude understanding of the upstream control of palmitoylating and depalmitoylating enzymes. The emerging evidence for palmitoylation cascades $[50,109]$, alongside an understanding of the importance of other post-translational modifications in the control of zDHHC-PAT activities and substrate interactions, holds promise for a clearer future.

Turning to the role of NCX1 palmitoylation in the control of calcium handling in the heart. Much like phosphorylation, our understanding of the importance of this regulatory mechanism will be supported by transgenic models in which palmitoylating enzymes are knocked out and overexpressed, as well as in more subtle knockin models targeting individual palmitoylation sites. The importance of understanding any contribution of aberrant palmitoylation to cardiac pathologies cannot be overstated, 
and may open the door to therapeutic strategies that target substrate recruitment by or catalytic activities of the individual palmitoylating and depalmitoylating enzymes.

\section{Competing Interests}

Declarations of interest: none

\section{CRediT roles}

Caglar Gök: Writing - original draft

William Fuller: Conceptualization; Writing - review \& editing.

\section{Acknowledgements}

We acknowledge financial support from the British Heart Foundation: SP/16/3/32317 to WF. 


\section{Figures}
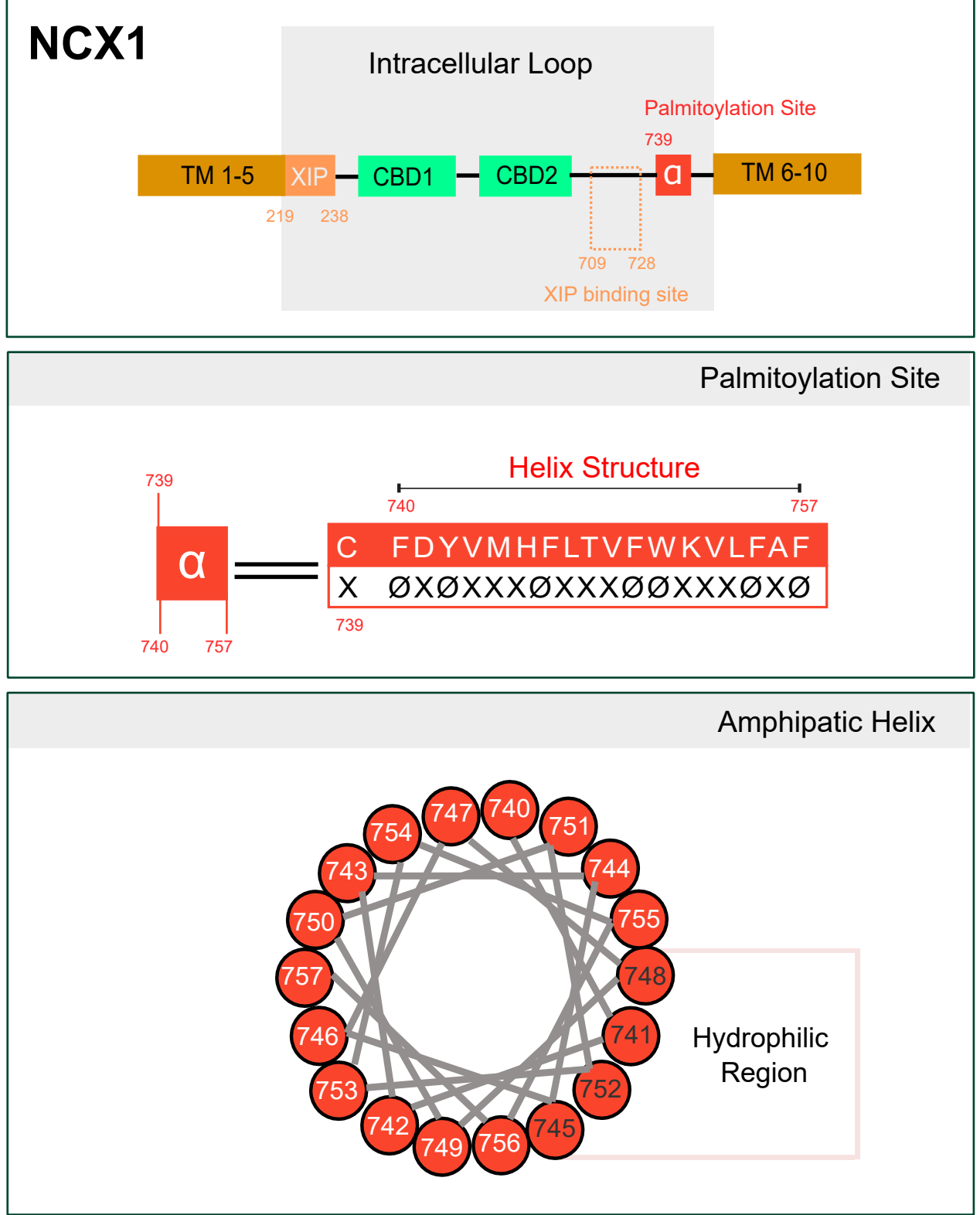

Figure 1: NCX1 schematic. The top panel shows full length NCX1, highlighting the positions of the transmembrane (TM) domains, and the large regulatory intracellular loop which includes the exchange inhibitory peptide (XIP), calcium binding domains (CBD) and palmitoylation site adjacent to the amphipathic $\alpha$ helix $(\alpha)$. The middle panel shows the sequence of the amphipathic helix, highlighting the enrichment of aromatic amino acids $(\varnothing)$ in this structure. The bottom panel shows the small hydrophilic face of this helix in a helical wheel projection. 


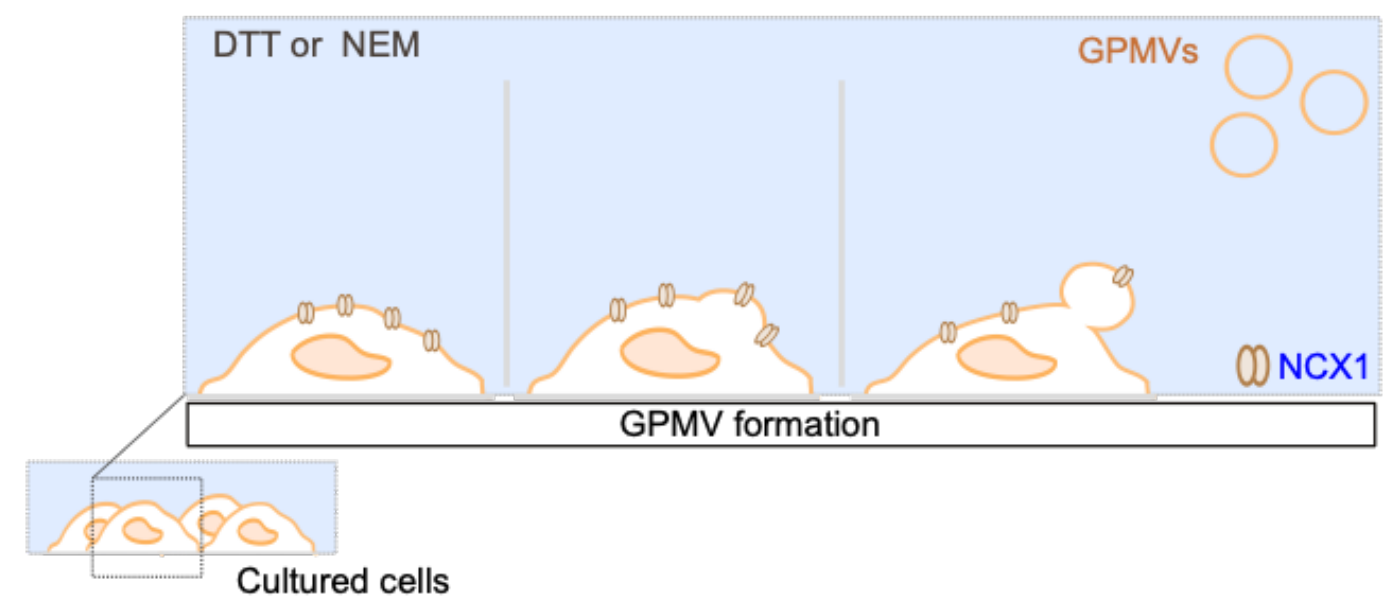

B

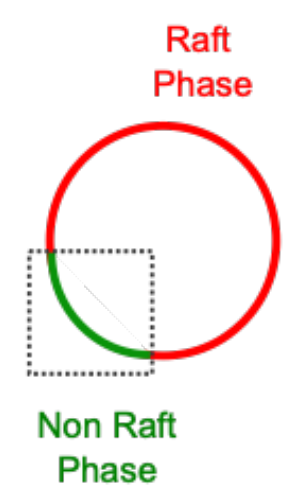

Phase Separated GPMV

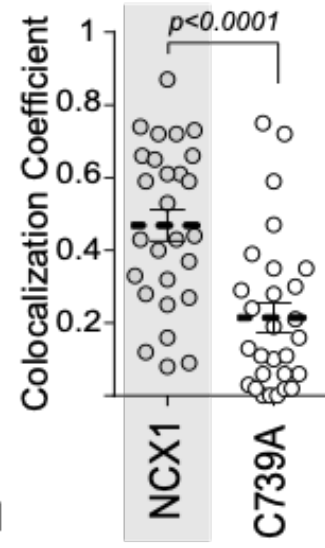

Raft / Non-Raft Colocalization

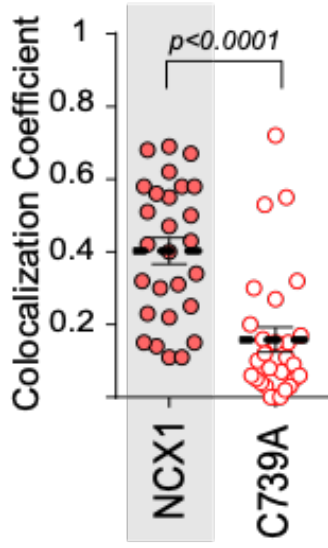

NCX1 / Raft Colocalization

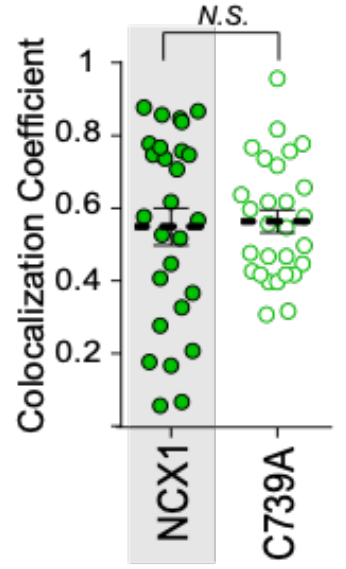

NCX1 / Non-Raft Colocalization

Figure 2: NCX1 behaviour in giant plasma membrane vesicles (GPMVs). A: Schematic of GPMV formation in the presence of vesiculation agents DTT or NEM. B: Schematic of a GPMV following phase separation into fluorescently labelled raft (red) and non-raft (green) phases. C: Increased colocalisation of raft and non-raft markers in the presence of WT versus C739A NCX1 suggests WT NCX1 impairs GPMV phase separation (Data adapted from Gök et al., 2020). D: Colocalisation of raft and non-raft markers with wild type and unpalmitoylatable NCX1 in phase-separated GPMVs. Left: increased raft colocalisation of WT versus C739A NCX1. Right: non-raft colocalisaton of wild type and C739A NCX1 (Data adapted from Gök et al., 2020). 


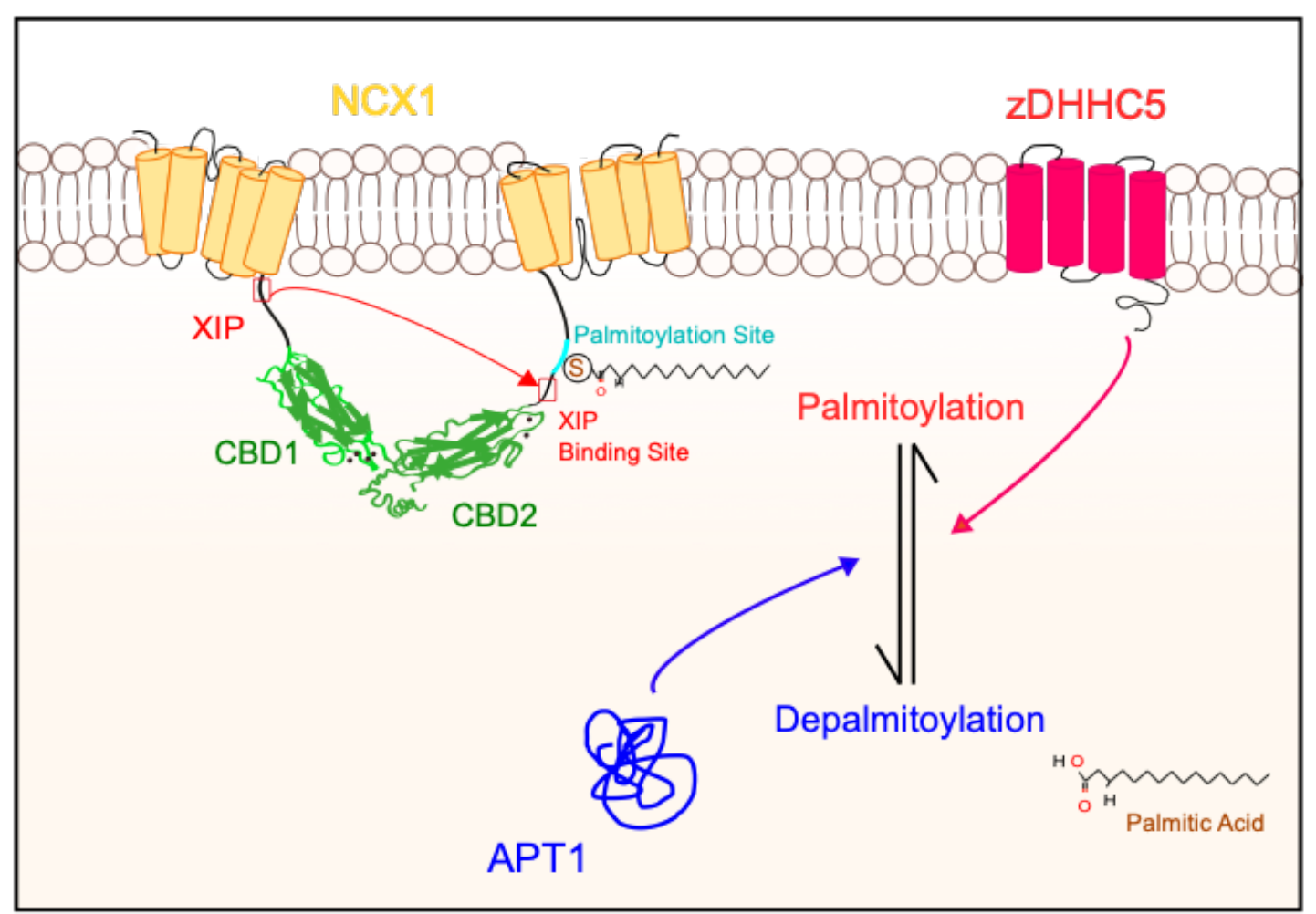

Figure 3: Illustration of NCX1 palmitoylation/ depalmitoylation cycle. NCX1 palmitoylation is catalyzed by zDHHC5 (magenta color); a surface-membrane resident zDHHC-PAT, and reversed by APT1 (navy blue). Palmitoylation enables XIP engagement with its binding site, which is located close to the NCX1 palmitoylation site. 


\section{References}

[1] M.E. Linder, R.J. Deschenes, Palmitoylation: policing protein stability and traffic, Nat Rev Mol Cell Biol 8(1) (2007) 74-84.

[2] J. Charollais, F.G. Van Der Goot, Palmitoylation of membrane proteins (Review), Mol Membr Biol 26(1) (2009) 55-66.

[3] Y. Fukata, M. Fukata, Protein palmitoylation in neuronal development and synaptic plasticity, Nat Rev Neurosci 11(3) (2010) 161-75.

[4] D.M. Bers, Calcium fluxes involved in control of cardiac myocyte contraction, Circ Res 87(4) (2000) 275-81.

[5] E.G. Lakatta, V.A. Maltsev, T.M. Vinogradova, A coupled SYSTEM of intracellular Ca2+ clocks and surface membrane voltage clocks controls the timekeeping mechanism of the heart's pacemaker, Circ Res 106(4) (2010) 659-73.

[6] M. Ohtsuka, H. Takano, M. Suzuki, Y. Zou, H. Akazawa, M. Tamagawa, K. Wakimoto, H. Nakaya, I. Komuro, Role of $\mathrm{Na}+\mathrm{Ca} 2+$ exchanger in myocardial ischemia/reperfusion injury: evaluation using a heterozygous $\mathrm{Na}+-\mathrm{Ca} 2+$ exchanger knockout mouse model, Biochem Biophys Res Commun 314(3) (2004) 849-53.

[7] M. Flesch, R.H. Schwinger, F. Schiffer, K. Frank, M. Sudkamp, F. Kuhn-Regnier, G. Arnold, M. Bohm, Evidence for functional relevance of an enhanced expression of the $\mathrm{Na}(+)-\mathrm{Ca} 2+$ exchanger in failing human myocardium, Circulation 94(5) (1996) 992-1002.

[8] N. Voigt, N. Li, Q. Wang, W. Wang, A.W. Trafford, I. Abu-Taha, Q. Sun, T. Wieland, U. Ravens, S. Nattel, X.H. Wehrens, D. Dobrev, Enhanced sarcoplasmic reticulum Ca2+ leak and increased Na+-Ca2+ exchanger function underlie delayed afterdepolarizations in patients with chronic atrial fibrillation, Circulation 125(17) (2012) 2059-70.

[9] W. Dai, B. Laforest, L. Tyan, K.M. Shen, R.D. Nadadur, F.J. Alvarado, S.R. Mazurek, S. Lazarevic, M. Gadek, Y. Wang, Y. Li, H.H. Valdivia, L. Shen, M.T. Broman, I.P. Moskowitz, C.R. Weber, A calcium transport mechanism for atrial fibrillation in Tbx5-mutant mice, eLife 8 (2019).

[10] Z. Li, D.A. Nicoll, A. Collins, D.W. Hilgemann, A.G. Filoteo, J.T. Penniston, J.N. Weiss, J.M. Tomich, K.D. Philipson, Identification of a peptide inhibitor of the cardiac sarcolemmal $\mathrm{Na}(+)-\mathrm{Ca} 2+$ exchanger, J Biol Chem 266(2) (1991) 1014-20.

[11] M. Ottolia, D.A. Nicoll, K.D. Philipson, Roles of two Ca2+-binding domains in regulation of the cardiac Na+-Ca2+ exchanger, J Biol Chem 284(47) (2009) 32735-41.

[12] S.A. John, B. Ribalet, J.N. Weiss, K.D. Philipson, M. Ottolia, Ca2+-dependent structural rearrangements within Na+-Ca2+ exchanger dimers, Proc Natl Acad Sci U S A 108(4) (2011) 1699-704.

[13] L. Reilly, J. Howie, K. Wypijewski, M.L. Ashford, D.W. Hilgemann, W. Fuller, Palmitoylation of the $\mathrm{Na} / \mathrm{Ca}$ exchanger cytoplasmic loop controls its inactivation and internalization during stress signaling, FASEB J 29(11) (2015) 4532-43.

[14] W. Fuller, L. Reilly, D.W. Hilgemann, S-palmitoylation and the regulation of NCX1, Channels (Austin) 10(2) (2016) 75-7.

[15] F. Plain, S.D. Congreve, R.S.Z. Yee, J. Kennedy, J. Howie, C.W. Kuo, N.J. Fraser, W. Fuller, An amphipathic alpha-helix directs palmitoylation of the large intracellular loop of the sodium/calcium exchanger, J Biol Chem 292(25) (2017) 10745-10752.

[16] F. Plain, D. Turnbull, N.J. Fraser, W. Fuller, Understanding the rules governing NCX1 palmitoylation, Channels (Austin) 11(5) (2017) 377-379.

[17] S. Matsuoka, D.A. Nicoll, Z. He, K.D. Philipson, Regulation of cardiac $\mathrm{Na}(+)-\mathrm{Ca} 2+$ exchanger by the endogenous XIP region, J Gen Physiol 109(2) (1997) 273-86.

[18] D.A. Nicoll, X. Ren, M. Ottolia, M. Phillips, A.R. Paredes, J. Abramson, K.D. Philipson, What we know about the structure of NCX1 and how it relates to its function, Ann N Y Acad Sci 1099 (2007) 1-6.

[19] D.W. Hilgemann, A. Collins, S. Matsuoka, Steady-state and dynamic properties of cardiac sodiumcalcium exchange. Secondary modulation by cytoplasmic calcium and ATP, J Gen Physiol 100(6) (1992) 933-61.

[20] C. Gok, W. Fuller, Regulation of NCX1 by palmitoylation, Cell Calcium 86 (2020) 102158.

[21] X. Ren, D.A. Nicoll, G. Galang, K.D. Philipson, Intermolecular cross-linking of Na+-Ca2+ exchanger proteins: evidence for dimer formation, Biochemistry 47(22) (2008) 6081-7.

[22] C. Gok, F. Plain, A.D. Robertson, J. Howie, G.S. Baillie, N.J. Fraser, W. Fuller, Dynamic Palmitoylation of the Sodium-Calcium Exchanger Modulates Its Structure, Affinity for Lipid-Ordered Domains, and Inhibition by XIP, Cell Rep 31(10) (2020) 107697. 
[23] T. Lanyon-Hogg, M. Faronato, R.A. Serwa, E.W. Tate, Dynamic Protein Acylation: New Substrates, Mechanisms, and Drug Targets, Trends Biochem Sci 42(7) (2017) 566-581.

[24] M.S. Rana, P. Kumar, C.J. Lee, R. Verardi, K.R. Rajashankar, A. Banerjee, Fatty acyl recognition and transfer by an integral membrane S-acyltransferase, Science 359(6372) (2018) eaao6326.

[25] J.F. Chase, P.K. Tubbs, Specific inhibition of mitochondrial fatty acid oxidation by 2-bromopalmitate and its coenzyme A and carnitine esters, Biochem J 129(1) (1972) 55-65.

[26] D. Davda, M.A. El Azzouny, C.T. Tom, J.L. Hernandez, J.D. Majmudar, R.T. Kennedy, B.R. Martin, Profiling targets of the irreversible palmitoylation inhibitor 2-bromopalmitate, ACS chemical biology 8(9) (2013) 1912-7.

[27] B. Zheng, M. DeRan, X. Li, X. Liao, M. Fukata, X. Wu, 2-Bromopalmitate analogues as activity-based probes to explore palmitoyl acyltransferases, J Am Chem Soc 135(19) (2013) 7082-5.

[28] M.P. Pedro, A.A. Vilcaes, V.M. Tomatis, R.G. Oliveira, G.A. Gomez, J.L. Daniotti, 2-Bromopalmitate reduces protein deacylation by inhibition of acyl-protein thioesterase enzymatic activities, PLoS One 8(10) (2013) e75232.

[29] J.H. Lorent, B. Diaz-Rohrer, X. Lin, K. Spring, A.A. Gorfe, K.R. Levental, I. Levental, Structural determinants and functional consequences of protein affinity for membrane rafts, Nature communications 8(1) (2017) 1219.

[30] X. Lin, A.A. Gorfe, I. Levental, Protein Partitioning into Ordered Membrane Domains: Insights from Simulations, Biophys J 114(8) (2018) 1936-1944.

[31] I. Levental, D. Lingwood, M. Grzybek, U. Coskun, K. Simons, Palmitoylation regulates raft affinity for the majority of integral raft proteins, Proc Natl Acad Sci U S A 107(51) (2010) 22050-4.

[32] J.T. Marinko, A.K. Kenworthy, C.R. Sanders, Peripheral myelin protein 22 preferentially partitions into ordered phase membrane domains, Proc Natl Acad Sci U S A 117(25) (2020) 14168-14177.

[33] E. Sezgin, H.J. Kaiser, T. Baumgart, P. Schwille, K. Simons, I. Levental, Elucidating membrane structure and protein behavior using giant plasma membrane vesicles, Nat Protoc 7(6) (2012) 1042-51. [34] Z. Gerstle, R. Desai, S.L. Veatch, Giant Plasma Membrane Vesicles: An Experimental Tool for Probing the Effects of Drugs and Other Conditions on Membrane Domain Stability, Methods Enzymol 603 (2018) $129-150$.

[35] Q. Liu, C. Bi, J. Li, X. Liu, R. Peng, C. Jin, Y. Sun, Y. Lyu, H. Liu, H. Wang, C. Luo, W. Tan, Generating Giant Membrane Vesicles from Live Cells with Preserved Cellular Properties, Research (Wash D C) 2019 (2019) 6523970.

[36] A.D. Skinkle, K.R. Levental, I. Levental, Cell-Derived Plasma Membrane Vesicles Are Permeable to Hydrophilic Macromolecules, Biophys J 118(6) (2020) 1292-1300.

[37] I. Levental, M. Grzybek, K. Simons, Raft domains of variable properties and compositions in plasma membrane vesicles, Proc Natl Acad Sci U S A 108(28) (2011) 11411-6.

[38] L. Janosi, Z. Li, J.F. Hancock, A.A. Gorfe, Organization, dynamics, and segregation of Ras nanoclusters in membrane domains, Proc Natl Acad Sci U S A 109(21) (2012) 8097-102.

[39] F. Sun, C.F.E. Schroer, C.R. Palacios, L. Xu, S.Z. Luo, S.J. Marrink, Molecular mechanism for bidirectional regulation of CD44 for lipid raft affiliation by palmitoylations and PIP2, PLoS Comput Biol 16(4) (2020) e1007777.

[40] R. Zidovetzki, I. Levitan, Use of cyclodextrins to manipulate plasma membrane cholesterol content: evidence, misconceptions and control strategies, Biochim Biophys Acta 1768(6) (2007) 1311-24.

[41] J. Kimura, A. Noma, H. Irisawa, Na-Ca exchange current in mammalian heart cells, Nature 319(6054) (1986) 596-7.

[42] D.W. Hilgemann, S. Matsuoka, G.A. Nagel, A. Collins, Steady-state and dynamic properties of cardiac sodium-calcium exchange. Sodium-dependent inactivation, J Gen Physiol 100(6) (1992) 905-32.

[43] D.A. Mitchell, A. Vasudevan, M.E. Linder, R.J. Deschenes, Protein palmitoylation by a family of DHHC protein S-acyltransferases, J Lipid Res 47(6) (2006) 1118-27.

[44] P. Strzyz, Sorting it out at the Golgi, Nat Rev Mol Cell Biol 20(1) (2019) 2-3.

[45] A.M. Ernst, S.A. Syed, O. Zaki, F. Bottanelli, H. Zheng, M. Hacke, Z. Xi, F. Rivera-Molina, M. Graham, A.A. Rebane, P. Bjorkholm, D. Baddeley, D. Toomre, F. Pincet, J.E. Rothman, S-Palmitoylation Sorts Membrane Cargo for Anterograde Transport in the Golgi, Dev Cell 47(4) (2018) 479-493 e7.

[46] A.M. Ernst, D. Toomre, J.S. Bogan, Acylation - A New Means to Control Traffic Through the Golgi, Front Cell Dev Biol 7 (2019) 109.

[47] O. Rocks, M. Gerauer, N. Vartak, S. Koch, Z.P. Huang, M. Pechlivanis, J. Kuhlmann, L. Brunsveld, A. Chandra, B. Ellinger, H. Waldmann, P.I. Bastiaens, The palmitoylation machinery is a spatially organizing system for peripheral membrane proteins, Cell 141(3) (2010) 458-71. 
[48] K. Lemonidis, O.A. Gorleku, M.C. Sanchez-Perez, C. Grefen, L.H. Chamberlain, The Golgi S-acylation machinery comprises $z \mathrm{DHHC}$ enzymes with major differences in substrate affinity and S-acylation activity, Mol Biol Cell 25(24) (2014) 3870-83.

[49] J. Howie, L. Reilly, N.J. Fraser, J.M. Vlachaki Walker, K.J. Wypijewski, M.L. Ashford, S.C. Calaghan, H. McClafferty, L. Tian, M.J. Shipston, A. Boguslavskyi, M.J. Shattock, W. Fuller, Substrate recognition by the cell surface palmitoyl transferase DHHC5, Proc Natl Acad Sci U S A (2014).

[50] F. Plain, J. Howie, J. Kennedy, E. Brown, M.J. Shattock, N.J. Fraser, W. Fuller, Control of protein palmitoylation by regulating substrate recruitment to a zDHHC-protein acyltransferase, Commun Biol 3(1) (2020) 411.

[51] K. Essandoh, J.M. Philippe, P.M. Jenkins, M.J. Brody, Palmitoylation: A Fatty Regulator of Myocardial Electrophysiology, Front Physiol 11 (2020) 108.

[52] Z. Pei, Y. Xiao, J. Meng, A. Hudmon, T.R. Cummins, Cardiac sodium channel palmitoylation regulates channel availability and myocyte excitability with implications for arrhythmia generation, Nature communications 7 (2016) 12035.

[53] J. Howie, L.B. Tulloch, M.J. Shattock, W. Fuller, Regulation of the cardiac Na(+) pump by palmitoylation of its catalytic and regulatory subunits, Biochem Soc Trans 41(1) (2013) 95-100.

[54] L.B. Tulloch, J. Howie, K.J. Wypijewski, C.R. Wilson, W.G. Bernard, M.J. Shattock, W. Fuller, The inhibitory effect of phospholemman on the sodium pump requires its palmitoylation, J Biol Chem 286(41) (2011) 36020-31.

[55] K. Takimoto, E.K. Yang, L. Conforti, Palmitoylation of KChIP splicing variants is required for efficient cell surface expression of Kv4.3 channels, J Biol Chem 277(30) (2002) 26904-11.

[56] A. Murthy, S.W. Workman, M. Jiang, J. Hu, I. Sifa, T. Bernas, W. Tang, I. Deschenes, G.N. Tseng, Dynamic palmitoylation regulates trafficking of $\mathrm{K}$ channel interacting protein 2 (KChIP2) across multiple subcellular compartments in cardiac myocytes, J Mol Cell Cardiol 135 (2019) 1-9.

[57] A.J. Chien, T. Gao, E. Perez-Reyes, M.M. Hosey, Membrane targeting of L-type calcium channels. Role of palmitoylation in the subcellular localization of the beta2a subunit, J Biol Chem 273(36) (1998) 23590-7.

[58] A.J. Chien, K.M. Carr, R.E. Shirokov, E. Rios, M.M. Hosey, Identification of palmitoylation sites within the L-type calcium channel beta2a subunit and effects on channel function, J Biol Chem 271(43) (1996) 26465-8.

[59] T. Zhou, J. Li, P. Zhao, H. Liu, D. Jia, H. Jia, L. He, Y. Cang, S. Boast, Y.H. Chen, H. Thibault, M. ScherrerCrosbie, S.P. Goff, B. Li, Palmitoyl acyltransferase Aph2 in cardiac function and the development of cardiomyopathy, Proc Natl Acad Sci U S A 112(51) (2015) 15666-71.

[60] J. Howie, K.J. Wypijewski, F. Plain, L.B. Tulloch, N.J. Fraser, W. Fuller, Greasing the wheels or a spanner in the works? Regulation of the cardiac sodium pump by palmitoylation, Crit Rev Biochem Mol Biol 53(2) (2018) 175-191.

[61] S. Lobo, W.K. Greentree, M.E. Linder, R.J. Deschenes, Identification of a Ras palmitoyltransferase in Saccharomyces cerevisiae, J Biol Chem 277(43) (2002) 41268-73.

[62] A.F. Roth, Y. Feng, L. Chen, N.G. Davis, The yeast DHHC cysteine-rich domain protein Akr1p is a palmitoyl transferase, J. Cell Biol. 159(1) (2002) 23-8.

[63] C.E. Ducker, E.M. Stettler, K.J. French, J.J. Upson, C.D. Smith, Huntingtin interacting protein 14 is an oncogenic human protein: palmitoyl acyltransferase, Oncogene 23(57) (2004) 9230-7.

[64] K. Huang, A. Yanai, R. Kang, P. Arstikaitis, R.R. Singaraja, M. Metzler, A. Mullard, B. Haigh, C. Gauthier-Campbell, C.A. Gutekunst, M.R. Hayden, A. El-Husseini, Huntingtin-interacting protein HIP14 is a palmitoyl transferase involved in palmitoylation and trafficking of multiple neuronal proteins, Neuron 44(6) (2004) 977-86.

[65] M. Fukata, Y. Fukata, H. Adesnik, R.A. Nicoll, D.S. Bredt, Identification of PSD-95 palmitoylating enzymes, Neuron 44(6) (2004) 987-96.

[66] C.A. Keller, X. Yuan, P. Panzanelli, M.L. Martin, M. Alldred, M. Sassoe-Pognetto, B. Luscher, The gamma2 subunit of GABA(A) receptors is a substrate for palmitoylation by GODZ, J Neurosci 24(26) (2004) 5881-91.

[67] C. Fernandez-Hernando, M. Fukata, P.N. Bernatchez, Y. Fukata, M.I. Lin, D.S. Bredt, W.C. Sessa, Identification of Golgi-localized acyl transferases that palmitoylate and regulate endothelial nitric oxide synthase, J. Cell Biol. 174(3) (2006) 369-77.

[68] J.T. Swarthout, S. Lobo, L. Farh, M.R. Croke, W.K. Greentree, R.J. Deschenes, M.E. Linder, DHHC9 and GCP16 constitute a human protein fatty acyltransferase with specificity for $\mathrm{H}$ - and N-Ras, J Biol Chem 280(35) (2005) 31141-8. 
[69] J.A. Duncan, A.G. Gilman, Characterization of Saccharomyces cerevisiae acyl-protein thioesterase 1 , the enzyme responsible for $G$ protein alpha subunit deacylation in vivo, J Biol Chem 277(35) (2002) 31740-52.

[70] J.A. Duncan, A.G. Gilman, A cytoplasmic acyl-protein thioesterase that removes palmitate from G protein alpha subunits and p21(RAS), J Biol Chem 273(25) (1998) 15830-7.

[71] E. Kong, S. Peng, G. Chandra, C. Sarkar, Z. Zhang, M.B. Bagh, A.B. Mukherjee, Dynamic palmitoylation links cytosol-membrane shuttling of acyl-protein thioesterase-1 and acyl-protein thioesterase- 2 with that of proto-oncogene $\mathrm{H}$-ras product and growth-associated protein-43, J Biol Chem 288(13) (2013) 9112-25.

[72] D.C. Yeh, J.A. Duncan, S. Yamashita, T. Michel, Depalmitoylation of endothelial nitric-oxide synthase by acyl-protein thioesterase 1 is potentiated by $\mathrm{Ca}(2+)$-calmodulin, J Biol Chem 274(46) (1999) 33148-54.

[73] L.A. Camp, S.L. Hofmann, Purification and properties of a palmitoyl-protein thioesterase that cleaves palmitate from H-Ras, J Biol Chem 268(30) (1993) 22566-74.

[74] A.A. Soyombo, S.L. Hofmann, Molecular cloning and expression of palmitoyl-protein thioesterase 2 (PPT2), a homolog of lysosomal palmitoyl-protein thioesterase with a distinct substrate specificity, J Biol Chem 272(43) (1997) 27456-63.

[75] P. Gupta, A.A. Soyombo, A. Atashband, K.E. Wisniewski, J.M. Shelton, J.A. Richardson, R.E. Hammer, S.L. Hofmann, Disruption of PPT1 or PPT2 causes neuronal ceroid lipofuscinosis in knockout mice, Proc Natl Acad Sci U S A 98(24) (2001) 13566-71.

[76] D.T. Lin, E. Conibear, ABHD17 proteins are novel protein depalmitoylases that regulate N-Ras palmitate turnover and subcellular localization, eLife 4 (2015) e11306.

[77] R.S. Kathayat, Y. Cao, P.D. Elvira, P.A. Sandoz, M.E. Zaballa, M.Z. Springer, L.E. Drake, K.F. Macleod, F.G. van der Goot, B.C. Dickinson, Active and dynamic mitochondrial S-depalmitoylation revealed by targeted fluorescent probes, Nature communications 9(1) (2018) 334.

[78] F.J. Dekker, O. Rocks, N. Vartak, S. Menninger, C. Hedberg, R. Balamurugan, S. Wetzel, S. Renner, M. Gerauer, B. Scholermann, M. Rusch, J.W. Kramer, D. Rauh, G.W. Coates, L. Brunsveld, P.I. Bastiaens, H. Waldmann, Small-molecule inhibition of APT1 affects Ras localization and signaling, Nat Chem Biol 6(6) (2010) 449-56.

[79] G. Siegel, G. Obernosterer, R. Fiore, M. Oehmen, S. Bicker, M. Christensen, S. Khudayberdiev, P.F. Leuschner, C.J. Busch, C. Kane, K. Hubel, F. Dekker, C. Hedberg, B. Rengarajan, C. Drepper, H. Waldmann, S. Kauppinen, M.E. Greenberg, A. Draguhn, M. Rehmsmeier, J. Martinez, G.M. Schratt, A functional screen implicates microRNA-138-dependent regulation of the depalmitoylation enzyme APT1 in dendritic spine morphogenesis, Nat Cell Biol 11(6) (2009) 705-16.

[80] N. Vartak, B. Papke, H.E. Grecco, L. Rossmannek, H. Waldmann, C. Hedberg, P.I. Bastiaens, The autodepalmitoylating activity of APT maintains the spatial organization of palmitoylated membrane proteins, Biophys J 106(1) (2014) 93-105.

[81] N. Adachi, D.T. Hess, P. McLaughlin, J.S. Stamler, S-Palmitoylation of a Novel Site in the beta2Adrenergic Receptor Associated with a Novel Intracellular Itinerary, J Biol Chem 291(38) (2016) 20232 46.

[82] S.J. Won, M. Cheung See Kit, B.R. Martin, Protein depalmitoylases, Crit Rev Biochem Mol Biol 53(1) (2018) 83-98.

[83] X. Wei, S. Adak, M. Zayed, L. Yin, C. Feng, S.L. Speck, R.S. Kathayat, Q. Zhang, B.C. Dickinson, C.F. Semenkovich, Endothelial Palmitoylation Cycling Coordinates Vessel Remodeling in Peripheral Artery Disease, Circ Res 127(2) (2020) 249-265.

[84] H. Sugimoto, H. Hayashi, S. Yamashita, Purification, cDNA cloning, and regulation of lysophospholipase from rat liver, J Biol Chem 271(13) (1996) 7705-11.

[85] Y. Cao, T. Qiu, R.S. Kathayat, S.A. Azizi, A.K. Thorne, D. Ahn, Y. Fukata, M. Fukata, P.A. Rice, B.C. Dickinson, ABHD10 is an S-depalmitoylase affecting redox homeostasis through peroxiredoxin-5, Nat Chem Biol 15(12) (2019) 1232-1240.

[86] K.J. Wypijewski, M. Tinti, W. Chen, D. Lamont, M.L. Ashford, S.C. Calaghan, W. Fuller, Identification of caveolar resident proteins in ventricular myocytes using a quantitative proteomic approach: dynamic changes in caveolar composition following adrenoceptor activation, Mol Cell Proteomics 14(3) (2015) 596-608.

[87] Y. Ohno, A. Kihara, T. Sano, Y. Igarashi, Intracellular localization and tissue-specific distribution of human and yeast DHHC cysteine-rich domain-containing proteins, Biochim Biophys Acta 1761(4) (2006) 474-83. 
[88] I. Dostanic, J. Schultz Jel, J.N. Lorenz, J.B. Lingrel, The alpha 1 isoform of Na,K-ATPase regulates cardiac contractility and functionally interacts and co-localizes with the $\mathrm{Na} / \mathrm{Ca}$ exchanger in heart, J Biol Chem 279(52) (2004) 54053-61.

[89] P.F. James, I.L. Grupp, G. Grupp, A.L. Woo, G.R. Askew, M.L. Croyle, R.A. Walsh, J.B. Lingrel, Identification of a specific role for the $\mathrm{Na}, \mathrm{K}$-ATPase alpha 2 isoform as a regulator of calcium in the heart, Mol. Cell 3(5) (1999) 555-63.

[90] S. Despa, J.B. Lingrel, D.M. Bers, Na/K-ATPase alpha2-isoform preferentially modulates Ca transients and sarcoplasmic reticulum Ca release in cardiac myocytes, Cardiovasc Res (2012).

[91] F.M. Lu, C. Deisl, D.W. Hilgemann, Profound regulation of $\mathrm{Na} / \mathrm{K}$ pump activity by transient elevations of cytoplasmic calcium in murine cardiac myocytes, elife 5 (2016).

[92] P.J. Ko, C. Woodrow, M.M. Dubreuil, B.R. Martin, R. Skouta, M.C. Bassik, S.J. Dixon, A ZDHHC5GOLGA7 Protein Acyltransferase Complex Promotes Nonapoptotic Cell Death, Cell Chem Biol 26(12) (2019) 1716-1724 e9.

[93] K.T. Woodley, M.O. Collins, S-acylated Golga7b stabilises DHHC5 at the plasma membrane to regulate cell adhesion, EMBO reports 20(10) (2019) e47472.

[94] B.C. Jennings, M.E. Linder, DHHC protein S-acyltransferases use similar ping-pong kinetic mechanisms but display different acyl-CoA specificities, J Biol Chem 287(10) (2012) 7236-45.

[95] K. Lemonidis, M.C. Sanchez-Perez, L.H. Chamberlain, Identification of a Novel Sequence Motif Recognized by the Ankyrin Repeat Domain of zDHHC17/13 S-Acyltransferases, J Biol Chem 290(36) (2015) 21939-50.

[96] N.J. Fraser, J. Howie, K.J. Wypijewski, W. Fuller, Therapeutic targeting of protein S-acylation for the treatment of disease, Biochem Soc Trans 48(1) (2020) 281-290.

[97] G.S. Brigidi, B. Santyr, J. Shimell, B. Jovellar, S.X. Bamji, Activity-regulated trafficking of the palmitoyl-acyl transferase DHHC5, Nature communications 6 (2015) 8200.

[98] M. Kohlhaas, T. Liu, A. Knopp, T. Zeller, M.F. Ong, M. Bohm, B. O'Rourke, C. Maack, Elevated cytosolic $\mathrm{Na}+$ increases mitochondrial formation of reactive oxygen species in failing cardiac myocytes, Circulation 121(14) (2010) 1606-13.

[99] C. Maack, S. Cortassa, M.A. Aon, A.N. Ganesan, T. Liu, B. O'Rourke, Elevated cytosolic Na+ decreases mitochondrial $\mathrm{Ca} 2+$ uptake during excitation-contraction coupling and impairs energetic adaptation in cardiac myocytes, Circ Res 99(2) (2006) 172-82.

[100] C. Maack, B. O'Rourke, Excitation-contraction coupling and mitochondrial energetics, Basic Res Cardiol 102(5) (2007) 369-92.

[101] T. Liu, B. O'Rourke, Enhancing mitochondrial Ca2+ uptake in myocytes from failing hearts restores energy supply and demand matching, Circ Res 103(3) (2008) 279-88.

[102] D. Aksentijević, A. Karlstaedt, M.V. Basalay, B.A. O’Brien, D. Sanchez-Tatay, S. Eminaga, A. Thakker, D.A. Tennant, W. Fuller, T.R. Eykyn, H. Taegtmeyer, M.J. Shattock, Intracellular sodium elevation reprograms cardiac metabolism, Nature communications 11 (2020) 4337.

[103] D. Aksentijevic, B.A. O'Brien, T.R. Eykyn, M.J. Shattock, Is there a causal link between intracellular Na elevation and metabolic remodelling in cardiac hypertrophy?, Biochem Soc Trans 46(4) (2018) 817827

[104] A. Boguslavskyi, D. Pavlovic, K. Aughton, J.E. Clark, J. Howie, W. Fuller, M.J. Shattock, Cardiac hypertrophy in mice expressing unphosphorylatable phospholemman, Cardiovasc Res 104(1) (2014) 72 82.

[105] S. Despa, M.A. Islam, C.R. Weber, S.M. Pogwizd, D.M. Bers, Intracellular $\mathrm{Na}(+)$ concentration is elevated in heart failure but $\mathrm{Na} / \mathrm{K}$ pump function is unchanged, Circulation 105(21) (2002) 2543-8.

[106] W. Fuller, L.B. Tulloch, M.J. Shattock, S.C. Calaghan, J. Howie, K.J. Wypijewski, Regulation of the cardiac sodium pump, Cell Mol Life Sci 70(8) (2013) 1357-80.

[107] C. Maack, A. Ganesan, A. Sidor, B. O'Rourke, Cardiac sodium-calcium exchanger is regulated by allosteric calcium and exchanger inhibitory peptide at distinct sites, Circ Res 96(1) (2005) 91-9.

[108] R.D. Kirkton, N. Bursac, Engineering biosynthetic excitable tissues from unexcitable cells for electrophysiological and cell therapy studies, Nature communications 2 (2011) 300.

[109] L. Abrami, T. Dallavilla, P.A. Sandoz, M. Demir, B. Kunz, G. Savoglidis, V. Hatzimanikatis, F.G. van der Goot, Identification and dynamics of the human ZDHHC16-ZDHHC6 palmitoylation cascade, eLife 6 (2017). 